\title{
Les drames conjugaux à la fin du XIXe siècle dans la «Chronique » de La Gazette des tribunaux
}

\section{Anne Durepaire}

\section{(2) OpenEdition}

\section{Édition électronique}

URL : http://journals.openedition.org/abpo/152

DOI : 10.4000/abpo.152

ISBN : 978-2-7535-1513-0

ISSN : $2108-6443$

\section{Éditeur}

Presses universitaires de Rennes

Édition imprimée

Date de publication : 1 mars 2009

Pagination : 89-98

ISBN : 978-2-7535-0875-0

ISSN : 0399-0826

\section{Référence électronique}

Anne Durepaire, «Les drames conjugaux à la fin du XIXe siècle dans la «Chronique » de La Gazette des tribunaux ", Annales de Bretagne et des Pays de l'Ouest [En ligne], 116-1 | 2009, mis en ligne le 01 mars 2011, consulté le 20 avril 2019. URL : http://journals.openedition.org/abpo/152 ; DOI : 10.4000/ abpo. 152 


\title{
Les drames conjugaux à la fin du XIX $x^{e}$ siècle dans la «Chronique » de La Gazette des tribunaux
}

\author{
Anne DUREPAIRE \\ Documentaliste \\ Doctorante en histoire contemporaine, \\ GERHICO-CERHILIM - université de Poitiers
}

Les faits divers possèdent cette caractéristique troublante et paradoxale, déjà évoquée par Michèle Perrot dans un article fondateur ${ }^{1}$, de donner l'impression, à celui qui les lit, d'une étrange répétition malgré l'apparence hétéroclite du matériau. II est vrai que si on les réduit à leur simple aspect factuel - " un homme tue sa femme » - l'éternité du crime l'emporte sur les particularités de la mise en scène. Or si les drames conjugaux peuvent apparaître comme un phénomène intemporel, ils sont bien, dans la manière de les présenter, tributaires d'une époque et d'un contexte éditorial.

La Gazette des Tribunaux, journal de jurisprudence et de débats judiciaires regroupe au sein d'une rubrique "Chronique » un ensemble de faits disparates qui n'ont pu trouver leur place dans les autres rubriques du quotidien, constituées par les décisions de jurisprudence et les débats judiciaires de la justice civile et criminelle. Au cours de la période étudiée, la structure de la «Chronique » est restée relativement stable. Les faits divers sont placés à la troisième page du quotidien qui en compte quatre (la quatrième page étant constituée des annonces légales), et chacun des faits divers occupe généralement un quart à une demi-colonne, alors que les débats judiciaires occupent au minimum une colonne, voire une et demie. Leur position finale situe les faits divers dans le prolongement des décisions et des débats judiciaires tout en constituant un espace libéré dans l'écriture et dans le style, allant du formalisme des "attendus » à la forme classique du compte rendu de procès. Ils permettent au rédacteur et au lecteur de sortir de l'espace strict du tribunal, dominé par l'autorité et la parole du juge. La liberté de

1. Perrot, Michelle, "Fait divers et histoire au XIXe siècle », Annales ESC, 1983, p. 911 919. 
ton et la liberté du contenu accordées à son rédacteur favorisent la présentation et la représentation de l'espace social et de ses tensions ${ }^{2}$.

Si l'on est attentif aux infractions subies ou commises par les femmes, il apparaît rapidement que les faits divers relatant les violences exercées sur le corps constituaient la majorité de ces récits. De fait, après comptage, il s'avère que ces violences constituent, au gré des années, entre 55 et $70 \%$ de ces faits divers ${ }^{3}$. On peut s'interroger sur la signification de ces données qui résultent de choix éditoriaux Toutefois, cette représentation quantitative a au moins le mérite de poser la question de l'effet que cette multitude pouvait avoir sur le lectorat et sur les intentions de la Rédaction. Le lecteur de la Gazette des Tribunaux de 1886 découvrait quotidiennement dans la "Chronique », une femme agressée ou responsable de violences. Celui de 1905 pouvait avoir le sentiment que les femmes étaient moins présentes puisqu'on ne trouve que deux mentions tous les cinq jours; celui de 1914 partageait les mêmes impressions que son prédécesseur. On ne rencontre guère d'évolution spectaculaire. Tout au plus, peut-on noter les changements qui affectent les récits de suicides et d'accidents. Les premiers sont en effet très nombreux à être rapportés en $1886^{4}$. Les récits d'accidents, notamment d'incendies mortels, sont eux aussi le propre de la chronique de l'année 1886, ils disparaissent ensuite pour faire place aux récits d'accidents de voiture dans les années suivantes. La diminution très sensible des faits divers entre 1886 et 1895 est le seul événement remarquable qui touche la "Chronique ». II convient toutefois de signaler que ce sont les violences à l'intérieur du couple qui sont le plus souvent présentées aux lecteurs, soit le quart des affaires ${ }^{5}$, donnant ainsi l'impression d'une sorte de guerre des sexes et de déchirement continu entre maris et femmes. Ce sont donc les logiques des drames conjugaux, telles qu'elles figurent dans la Gazette des tribunaux, et le "repérage » des stéréotypes qui constituent les visées de la présente contribution.

2. À l'appui de cette démonstration, on pourra noter que c'est dans cet espace que Jules Moineaux et son célèbre fils, Georges Moineaux dit Courteline, ont déployé une partie de leur talent de chroniqueurs et de caricaturistes.

3. Une des particularités de la "Chronique » de La Gazette des tribunaux est de suivre parfois sur plusieurs numéros les avancées d'une enquête ou les audiences d'un procès. Il faut donc pour celui qui veut suivre de manière quantitative ces faits divers, faire la part entre le nombre de récits publiés et le nombre d'affaires effectivement traitées. On doit noter qu'en valeur absolue ou en valeur relative, la proportion des récits de violences reste la même, entre 55 et $70 \%$. On trouve ainsi pour chacune des années traitées pour cette étude : 1886 : 376 affaires sur 538 (424 sur 603 récits publiés), 1895, 102 affaires sur 184 (124/223), 1905, 108 affaires sur 180 (138/180), août 1913-juillet 1914: 100 affaires sur $151(111 / 170)$.

4. 29 affaires en 1886 contre 1 en 1895, 1 en 1905.

5. On compte 150 affaires sur 538 en 1886, 46 sur 184 en 1895, 43 sur 180 en 1905 et 42 sur 151 entre août 1913-juillet 1914. 
Jalousie et alcool : les « aiguillons de la violence conjugale »

« Un drame conjugal qui a vivement ému la population s'est déroulé jeudi matin à Dunkerque. Un douanier, $\mathrm{M}$. Lebreton caserné à la Tente Verte, après une violente discussion avec sa femme, lui a tiré trois coups de revolver. Les projectiles blessèrent mortellement la malheureuse à la tête et à la poitrine. Le premier, tournant alors son arme contre lui-même, s'est fait sauter la cervelle. La mort a été foudroyante. Ce terrible drame est attribué à la jalousie ${ }^{6} . »$

Ce sont les querelles quotidiennes qui constituent la première toile de fond des violences conjugales. En 1886, Marie-Adrienne Virot est tuée de trois coups de revolver par son mari pris "dans une fureur jalouse ${ }^{7}$ ". M. C..., à la suite d'un accès de jalousie furieuse, décharge son revolver sur sa femme ${ }^{8}$. Et c'est vers 5 h 30 que le mari de Maria-Rosalie Legrand «à la suite d'une violente discussion, dans un moment de furie bestiale » la tue de deux coups de couteau, acte que la Gazette attribue aux « querelles fréquentes dues à la jalousie » de cette femme 9 . II ne faut pas voir dans le motif de la jalousie la réalité d'un adultère effectif; il n'est bien souvent que le sentiment inquiet d'une incertitude sur la fidélité du conjoint, attisé par le soupçon comme dans le cas du sieur Foucault, "mari violent et jaloux » qui soupçonne sa femme, domestique, d'avoir des relations avec son maître; pour son geste violent contre elle, il est traduit devant la justice correctionnelle ${ }^{10}$. Il existe aussi des natures jalouses exacerbées par le comportement $d^{\prime}$ ' une femme coquette et très courtisée ». Ces soupçons répondent à l'extrême "sensibilité » qui existe à cette époque autour de l'adultère. En effet, il constitue autant une faute morale qu'une faute que le droit reconnaît puisqu'elle ouvre au conjoint la possibilité de demander à la justice sa répression et d'intenter, depuis 1884 , une action en divorce.

En cour d'assises certains hommes évoquent l'ivresse, prétextant que l'alcool a exacerbé leur jalousie. Ils espèrent ainsi échapper à la justice ou à une lourde sanction. Toutefois, les auteurs de la «Chronique » s'en inquiètent et signalent qu'« il serait curieux et peut-être même effrayant de faire le relevé des crimes dont l'alcoolisme est la première cause. Maumy est lui aussi un alcoolique; la boisson ne produit pas sur lui une ivresse plus ou moins gaie, mais lui donne des accès de fureur terribles dont sa femme est la victime. Celle-ci avait même dû s'entendre avec sa logeuse pour éviter les brutalités de son mari; elle allait souvent dans une pièce inoccupée ${ }^{11}$ ". Ivrogne, querelleur et brutal, alcoolique violent, alcoolique invétéré sont autant de qualificatifs désignant les hommes violents. Lorsqu'une rixe conjugale éclate, les parents et les voisins sont souvent le «dernier refuge»

6. La Gazette des Tribunaux, 3 août 1895, Chronique, départements, Nord, Dunkerque.

7. La Gazette des Tribunaux, $1^{\text {er }}$ décembre 1886, Chronique, départements, Var, Toulon.

8. La Gazette des Tribunaux, 28 avril 1886, Chronique, Paris.

9. La Gazette des Tribunaux, 20 juin 1886, Chronique, Paris.

10. La Gazette des Tribunaux, 8 mars 1886, Chronique, Paris.

11. La Gazette des Tribunaux, 9 janvier 1895, Chronique, Paris. 
pour les victimes. Mais pour madame Gsell aucun lieu ne s'avère sûr. En 1914, tandis que son mari alcoolique lui faisait vivre "un véritable martyr ", elle quitte son ménage et se réfugie chez ses parents. Son mari la rejoint, la prie de revenir avec lui, puis il l'assassine de deux coups de revolver ${ }^{12}$. Cette situation, qui n'est pas unique, explique que parents, enfants, voisins, concierges sont parfois les victimes "collatérales» de ces drames conjugaux lorsqu'ils cherchent à s'interposer. Ainsi, en 1886, la dame Maillefer est tuée avec sa fille d'un coup de canne à épée par son gendre, employé des chemins de fer, venu se quereller avec sa femme qui s'était réfugiée chez sa mère depuis leur séparation ${ }^{13}$. Ce n'est pas la belle-mère mais le beaupère qu'Hippolyte-François Bellavoine, "alcoolique au caractère brutal ", frappe d'un coup de hache alors qu'il cherchait sa femme ${ }^{14}$. En 1895, la dame Thiers, "femme battue ", avait quitté son mari pour aller vivre dans un hôtel. Lorsque son époux surgit, c'est sur "la dame Castan, tenancière ", qu'il tire alors que cette dernière cherchait à calmer l'intrus ${ }^{15}$.

On peut se demander de quelle manière les lecteurs réagissaient à la lecture de ces violences. La répétition de faits divers relatifs aux « drames conjugaux » contribuait-t-elle à former le goût des lecteurs, à les accoutumer à la brutalité des maris? Toujours est-il que certaines scènes sont décrites froidement, mais ne parviennent pas à masquer le déchaînement de la violence, comme dans le cas de l'affaire Evrat. Le portrait du mari est peu flatteur : "II ne travaillait pas et buvait beaucoup. II frappait également sa femme, blanchisseuse, obligée de lui donner tout l'argent qu'elle gagnait. » Au lendemain de son crime, Evrat déclare qu'il a agi sous l'empire de l'ivresse, mais aussi de la jalousie: sa femme lui aurait annoncé qu'elle allait le quitter et vivre avec un autre homme pour se soustraire à ses mauvais traitements. Le lecteur peut imaginer aisément avec cette description, courte et concise, la scène du crime, son horreur. Un voisin remarque un filet de sang coulant sous la porte des époux; il s'empresse d'aller prévenir les agents du commissariat de police. Puis les agents font «sauter» la porte, car elle était fermée de l'intérieur; ils découvrent le meurtrier, ivre, endormi sur le sol, les mains ensanglantées. La description de la victime laisse imaginer que le chroniqueur a eu accès aux premières constatations médicales: "Elle porte une blessure au cou, et de multiples blessures sur le corps faites avec un tranchet et une hachette; sa tête a été broyée ${ }^{16}$. »

Associé à la jalousie et/ou à l'alcool, le crime apparaît comme le moment paroxysmique mais imprévisible où la colère d'un individu déborde, et où la violence se déchaîne, parfois à la limite de la folie. Les sentiments trop vifs, comme la jalousie ou les moments particuliers où on se laisse " griser » par l'alcool, apparaissent comme favorisant le passage à l'acte. Pour les

12. La Gazette des Tribunaux, 28 janvier 1914, Chronique, Paris.

13. La Gazette des Tribunaux, 17 janvier 1886, Chronique Paris.

14. La Gazette des Tribunaux, 2 octobre 1886, Chronique, Paris.

15. La Gazette des Tribunaux, 30 septembre 1895, Chronique, Paris.

16. La Gazette des Tribunaux, 20 janvier 1886, Chronique, Paris. 
rédacteurs de La Gazette des tribunaux, comme pour les lecteurs, c'est une façon privilégiée d'observer les violences à l'intérieur du couple. Ces récits comportent aussi une morale : il faut se satisfaire de "passions tempérées » et ne céder ni à son humeur ni à l'ivresse.

\section{Les drames de la rupture conjugale}

Sans doute, le trait le plus marquant des faits divers est qu'une forte proportion des drames conjugaux intervient dans des couples qui sont en rupture.

«Monery [...] était trompé par sa femme. Celle-ci, de mœurs légères, s'était éprise d'un maréchal-ferrant du voisinage. Un beau-jour, elle s'enfuit du domicile conjugal en emportant linge et argent. Monery acheta un revolver et le 6 décembre 1905 voyant les deux amants installés dans un restaurant du boulevard de Reuilly, il tira sur eux à plusieurs reprises. L'homme fut frappé à mort, la femme plus légèrement blessée. L'histoire est banale, hélas ${ }^{17}$ !»

Derrière ces crimes de sang se trouvent souvent des "drames de la séparation », ce sont des femmes qui se sont lassées des querelles, des jalousies, des brutalités. Mais ce sont aussi des femmes qui ont abandonné le domicile conjugal pour suivre un amant, cas banal d'adultère, ou " pour vivre en concubinage avec un autre homme ", et ainsi quitter définitivement son mari, mais sans divorcer ${ }^{18}$. Les hommes ne sont pas en reste. Le geste de la "femme Colinet " l'illustre : elle tire sur son mari « des coups de revolver car il l'avait abandonné pour vivre avec une de ses anciennes maîtresses ${ }^{19}$ ", de même Marie Bader qui tente de tuer son mari. En effet, mariée à un "homme qui ne dédaigne pas s'amuser» et qui lui a "signifié son congé et l'a invité à se placer comme domestique», elle ne peut supporter cette humiliation ${ }^{20}$. De son côté, la femme Tabart, née Louise Perrin, fleuriste de 27 ans, tue son mari par vengeance : il allait obtenir le divorce contre elle ${ }^{21}$.

Le palais de justice, lieu de conciliation, d'arbitrage ou de régulation, est aussi l'espace de "tragédies sanglantes »: "C'est un drame du divorce qui amène aujourd'hui la Cour d'Assises l'accusé Lénoard Plazenet, âgé de 49 ans, garçon de chais. » En effet, madame Plazenet « se proposait » de demander le divorce. Le 11 juillet dernier, les deux époux étaient convoqués au palais de justice pour la tentative de conciliation. En arrivant, $\mathrm{M}^{\mathrm{me}}$ Plazenet dit au garçon de bureau :

"j'ai peur que mon mari me fasse un mauvais coup. La comparution devant le président se passa sans incident. $\mathrm{M}^{\mathrm{me}}$ Plazenet sortit du palais par

17. La Gazette des Tribunaux, 18 décembre 1905, Chronique, Paris.

18. La Gazette des Tribunaux, 18 août 1886, Chronique, Paris.

19. La Gazette des Tribunaux, 6 décembre 1886, Chronique, Paris.

20. La Gazette des Tribunaux, 22 avril 1914, Chronique, Paris.

21. La Gazette des Tribunaux, 9 août 1886, Chronique, Paris. 
la place Dauphine. Son mari la suivit sur le quai de l'Horloge où elle s'engagea. Elle se mit à courir pour lui échapper mais il s'élança à sa poursuite. Un taxi-auto le renversa. II se releva brandissant son revolver, le déchargea à plusieurs reprises sur sa femme. Celle-ci tomba en face de la conciergerie. Plazenet continua à tirer sur elle et seule l'intervention d'un agent pu l'arrêter ${ }^{22}$.»

Le cas n'est pas isolé et le lecteur en découvre régulièrement de semblables. Quelques jours plus tôt, la Gazette rapportait l'aventure de " l'artistedécorateur bien connu ", M. Paul Mezzara. Alors qu'il se rendait accompagné de son avocat à la $4^{\mathrm{e}}$ chambre du tribunal civil de la Seine où son divorce allait être plaidé et qu'il traversait la salle des pas perdus, il entendit courir derrière lui : "II se retourna et vit sa femme qui, un revolver au poing, le menaçait. Mais dès qu'il fut retourné, celle-ci prit la fuite et elle avait quitté le palais avant que l'artiste fût revenu de sa stupeur ${ }^{23}$. »

Dans ces drames du divorce, c'est bien l'exaspération et la rancune nées de la rupture qui concourent au geste tragique. On retrouve les mêmes causes et les mêmes procédés lorsque ces femmes refusent ou persistent à refuser, malgré l'abandon du domicile conjugal, malgré l'inconduite éventuelle, de réintégrer le foyer conjugal. Les maris délaissés, puis abandonnés, n'en continuent pas moins à "chercher querelle » à leurs femmes, à "les poursuivre de leurs réclamations ». L'amour bafoué, l'honneur flétri expliquent en partie ces conduites masculines. La rupture est vécue comme une véritable injure qui demande réparation. L'affaire Delabert en est un exemple:

«Delabert a épousé en 1910, une jeune fille de 16 ans. Le ménage n'eut pas d'enfants et la femme ne tarda pas à se livrer à la prostitution puis à abandonner le domicile conjugal. Le 15 août 1913, Delabart qui voyait de temps en temps sa femme la supplia de reprendre la vie commune et la fit même admonester par le commissaire de police de son quartier. Le 24 août, les deux époux passèrent la nuit ensemble. Le lendemain, Delabart se constituait prisonnier en déclarant qu'il avait tué sa femme. Cette malheureuse fut trouvée chez lui, la gorge coupée, et portant la trace de neuf blessures. Delabart l'avait frappée à coups de couteau avec une férocité inouïe et lui avait tranché la carotide alors qu'elle était déjà grièvement blessée et dans l'impossibilité de se défendre. Delabart allègue qu'il a frappé sa femme parce qu'elle refusait d'abandonner sa vie irrégulière et l'avait insulté ${ }^{24}$. »

Condamné à deux années de prison par la cour d'assises de la Seine, Delabert n'a pas bénéficié totalement de «la jurisprudence devenue semble-t-il constante en matière de crimes passionnels » affirme le correspondant du journal judiciaire. Un autre chroniqueur anonyme de La Gazette des tribunaux fait part de circonstances presque identiques qui débouchent sur l'acquittement d'un dénommé Clairet. Celui-ci avait tiré en direction de sa femme cinq coups de revolver, sans l'atteindre il est vrai. Elle avait quitté le

22. La Gazette des Tribunaux, 19 novembre 1913, Chronique, Paris.

23. La Gazette des Tribunaux, 15 novembre 1913, Chronique, Paris.

24. La Gazette des Tribunaux, 17 novembre 1913, Chronique, Paris. 
domicile conjugal après de violentes scènes de ménage pour aller se placer chez un médecin des environs de Vichy. Malgré des lettres passionnées et menaçantes la suppliant de rentrer au foyer conjugal, «Marie, reviens, évite un malheur », elle avait refusé.

Le 27 octobre 1892, La Gazette des Tribunaux ne se contente pas de rapporter des faits divers ou des procès. Elle propose à ses lecteurs un commentaire de l'ouvrage d'Henri Coulon, Le divorce et l'adultère. De l'abrogation des lois pénales en matière d'adultère. Son auteur, avocat à la cour d'appel, actif défenseur du divorce par consentement mutuel, demande l'abrogation de l'article 336 du Code pénal qui permet au mari de dénoncer l'adultère de sa femme pour en obtenir la répression, des articles 337 et 338 qui punissent d'emprisonnement la femme coupable et son complice, et de l'article 339 qui frappe d'une amende le mari ayant entretenu une concubine au domicile conjugal, et excuse le meurtre commis par un mari sur sa femme et le complice de celle-ci, selon la thèse qui voudrait que depuis le rétablissement du divorce en 1884 , I'adultère n'est plus un délit mais uniquement une cause de divorce. Pour Henri Coulon, l'existence du divorce permet justement d'éviter les drames consécutifs à l'adultère et d'empêcher les assassinats. Le chroniqueur se transforme en critique judiciaire et ne cache pas aux lecteurs son hostilité à l'égard des thèses défendues. Il tente d'anéantir le raisonnement présenté et reprend à son compte le système établi depuis longtemps par Jules Simon :

"On prête ce raisonnement au mari outragé : "Puisqu'elle me trahit, je vais m'adresser aux tribunaux qui nous remettront l'un et l'autre dans l'état où nous étions l'un et l'autre avant le mariage et tout sera dit." Mais quel est l'homme qui parle ainsi, c'est un calculateur, un sage, ni accessible à la colère, ni à la jalousie. Celui-là n'assassinera point. Ce n'est pas à son bénéfice qu'on a découvert l'excuse du "crime passionnel". Mais mettez en lui la passion : supposez-le amoureux et jaloux jusqu'à la violence. Croyez-vous au moment où il aperçoit sous les yeux la preuve flagrante de l'adultère, il va se calmer subitement en disant : "j'ai le divorce"? »

Dans son système, l'excuse du crime d'adultère est reconnue ainsi que l'existence, dans le domaine de l'espace conjugal, d'une justice privée qui ne saurait être remplacée en ce domaine par la décision d'un tribunal. Et il y a aussi la reconnaissance de l'impuissance humaine à contenir "l'indignation et la colère » dans certaines circonstances de la vie intime, avec laquelle on peut tracer un parallèle avec tous ces accès qui peuplent les drames conjugaux dans ses querelles et ses ruptures, colère, jalousie, fureur.

\section{Faux mariages et femmes entretenues}

Si nous élargissons l'étude des conflits conjugaux aux autres formes de relations entre amants et maîtresses, les mêmes violences se retrouvent :

«Bondon vivait en concubinage depuis 17 ans avec une fille Carré. Quatre enfants leur étaient nés, deux vivent aujourd'hui. Tous les dimanches et quelquefois dans le courant de la semaine, Bondon et sa maîtresse 
s'enivraient. C'était alors des disputes et souvent des coups. Le 6 janvier dernier, ils étaient tous les deux pris de boisson, un marchand de vin nommé Delprat les entendant se quereller comme d'habitude, ne s'en inquiéta. Tout à coup, Bondon parut chez lui les traits bouleversés: "je crois, lui cria-t-il, que je viens de tuer ma femme." »

À l'audience, il pleure et explique qu'au cours d'une dispute, sa maîtresse lui avait donné plusieurs coups de pied et que poussé à bout, il l'avait frappé d'un coup de couteau qu'il tenait à la main sans se rendre compte de ce qu'il faisait.

«Vous vous enivrez souvent? lui dit M. le Président - Oh, répondit Bondon, mon Dieu, oui, comme tous les ouvriers. »

L'union libre n'empêche pas la jalousie des amants ni ne prévient les drames sanglants. Les couples, indépendamment de leur statut juridique, se ressemblent. En 1886, une jeune femme meurt étranglée par son amant : celui-ci «quant il est ivre devient horriblement jaloux ${ }^{25}$ ». Une autre jeune femme, "la fille Félicia », domestique, est tuée par son amant, un conseiller municipal, parce qu'il croyait qu'elle entretenait des relations avec une autre domestique ${ }^{26}$. Quant à la fille Eulalie Rassas, 23 ans, c'est parce qu'elle avait un deuxième amant que son premier amant la tue d'un coup de couteau en plein cœur ${ }^{27}$. En 1895, c'est une femme Pierson qui meurt étranglée : son amant, "extrêmement jaloux lui fait des scènes croyant qu'elle le trompe et qu'elle ne l'aime plus ${ }^{28} »$. Pour les lecteurs, nul doute que ce geste vindicatif est le plus souvent masculin et que les victimes sont généralement des femmes.

La rupture et l'abandon provoquent les mêmes sentiments de vengeance. Reste alors le dernier des recours, une forme de chantage ou de gestes conduisant à l'irréparable. Quelques femmes, bravant l'opinion des uns et des autres, s'adressent à la justice pour prévenir une escalade possible et mettre fin à une relation délétère. Un jeune homme est ainsi traduit en justice sous la prévention de menaces de mort contre une jeune blanchisseuse. À l'audience, il déclare " qu'il l'aimait bien, qu'il l'aimait trop pour lui faire du mal, qu'il voulait lui faire peur pour qu'elle revienne avec lui ». Le président fait un commentaire à voix haute et souligne que "c'est un singulier moyen ». La victime trouve des excuses à la conduite de son ancien compagnon : "Il dit la vérité, il m'aimait bien mais il était trop violent dans son amour. »

Dans les faits divers relatifs au couple, un élément, qui n'existait pas jusque-là, fait son apparition dans les colonnes du journal, le mariage de I'un des partenaires avec une autre femme ou un autre homme. Ainsi, alors qu'ils sont amants depuis quelques années, une jeune veuve refuse de se

25. La Gazette des Tribunaux, 22 mars 1886, Chronique, Paris.

26. La Gazette des Tribunaux, 3 septembre 1886, Chronique, départements, Calvados.

27. La Gazette des Tribunaux, 11 août 1886, Chronique, départements, Bouches-du-Rhône.

28. La Gazette des Tribunaux, 11 octobre 1895, Chronique, Paris. 
marier avec celui qui partage son existence. Alors, "dans un accès de rage » lors d'une ultime demande, son amant tente de la tuer ${ }^{29}$. Pour les mêmes raisons, un cuisinier de 23 ans tue sa maîtresse Joséphine de deux coups de couteau, avant de retourner l'arme contre lui : "Charles et Joséphine étaient liés depuis plusieurs années » et devaient se marier. Depuis près d'un mois, elle était venue à Marmande au service du propriétaire de la "buvette Arthur». Le cuisinier se présente "dimanche dernier à cet établissement : Joséphine Le Turc refuse de rentrer à Bordeaux. Elle ne voulait plus donner suite à leur projet de mariage ». Des menaces sont proférées et «le commissaire appela les deux amants dans son cabinet: Patier parut à ce magistrat agir sous l'empire d'une grande jalousie». Promesse fut faite devant le commissaire par Joséphine de donner suite au projet de mariage et, le temps de gagner quelque argent, de rentrer à Bordeaux. Le chroniqueur ajoute: "Patier rentrait à Marmande dès mardi soir (on le vit rôdant près de la buvette). Jeudi après-midi, Joséphine le rencontre. II a sans doute renouvelé ses supplications et qu'il supposait qu'elle avait un amant. Exaspéré par ses refus, il s'est jeté sur elle. »

Le désir de vengeance est aussi un sentiment éprouvé par les maîtresses délaissées. Victorine, âgée de 22 ans, a jeté au visage de son amant du vitriol car il lui avait signifié que leurs relations devaient cesser puisqu'il allait se marier ${ }^{30}$. Les femmes non mariées sont même deux fois plus nombreuses que les épouses à agir dans ces circonstances. Les logiques de la vengeance sont évidentes lorsque l'abandon a laissé la femme seule, enceinte ou avec des enfants; mais toutes ne passent pas à l'acte. Ainsi, Mlle Magnable tire sur son ancien amant trois coups de revolver avant de retourner l'arme contre elle : après lui avoir promis le mariage alors qu'elle était enceinte, il l'avait abandonnée et s'était fiancée avec une autre ${ }^{31}$. Une jeune domestique de 22 ans jette une grande quantité de vitriol à la figure de son amant: il l'avait quittée en la laissant seule avec trois enfants ${ }^{32}$. Quelques mois plus tard, c'est encore une jeune domestique qui lance du vitriol sur son amant : il l'avait abandonnée après l'avoir rendue mère ${ }^{33}$.

Il existe encore une autre catégorie de femmes dont on parle peu : les femmes entretenues, parfois à la limite de la prostitution, vivant de la galanterie et se faisant entretenir par un homme. Au fil de quelques faits divers, on suit leur parcours et leurs déboires: "Cécile Adam vivait modestement à Arcachon auprès de ses parents. " Sa sœur Marthe, plus âgée qu'elle, habitait depuis longtemps à Paris. De temps en temps, elle revenait au pays. Ses toilettes, les récits de ses distractions et des splendeurs du milieu desquelles elle vivait, éblouirent sa jeune sœur. Celle-ci, un beau jour, «abandonna sa cotte et ses sabots et débarqua à Paris ». Tout d'abord, elle chercha à

29. La Gazette des Tribunaux, 16 septembre 1886, Chronique, départements, Somme.

30. La Gazette des Tribunaux, 29 décembre 1895, Chronique, Paris.

31. La Gazette des Tribunaux, 18 septembre 1895, Chronique, Paris.

32. La Gazette des Tribunaux, 17 avril 1886, Chronique, Paris.

33. La Gazette des Tribunaux, 21 août 1886, Chronique, Paris. 
travailler, mais la vie fut dure : «Sa sœur lui prêta des toilettes et la promena avec elle dans les lieux publics. Elle eut un amant puis deux et vécut de la vie ordinaire des femmes galantes. » En 1894, elle fit la connaissance de «M. Saujot qui dirige le magasin de fleurs Vaillant-Rouzeau, boulevard des Capucines. Celui-ci subvenait à ses besoins et lui laissait une grande liberté dont Cécile Adam ne manquait pas d'user. Au commencement de l'année, M. S déclara qu'il allait la quitter. Cécile Adam ne l'entendait pas ainsi. Très dépensière, elle était toujours sans ressources et le départ de son amant allait augmenter son embarras. Au mois de février, très pressée d'argent, elle s'acharna à la poursuite de M. S. ${ }^{34}$ ».

La diversité des âges des protagonistes, les situations professionnelles et maritales, la durée des relations sont très variées : le point commun entre tous ces faits divers reste la rupture considérée comme inacceptable pour l'un des deux partenaires. Toutefois, lorsque les couples sont illégitimes ou que leurs liens n'ont pas été scellés par un mariage, les porte-parole de l'institution judiciaire prennent parfois leurs distances et considèrent que la séparation n'est pas forcément condamnable, même sur le plan moral. Un magistrat ironise même : "Si les unions libres ne pouvaient jamais être rompues, elles n'auraient aucun avantage sur le mariage bien au contraire. » C'est ainsi que s'exprime le président de la huitième chambre correctionnelle avant d'interroger une jeune femme de vingt ans poursuivie devant le tribunal pour coups et blessures ${ }^{35}$.

Au final, le lecteur du XIX $x^{e}$ siècle pourrait être surpris du nombre réduit d'éléments autour desquels s'articulent les violences au sein des couples. On aurait pu imaginer que les querelles conjugales naissent de «causalités » moins restreintes que I'alcool, la jalousie ou l'abandon. Les faits divers exposés aux lecteurs présentent les relations conjugales comme un huisclos fragile, un face-à-face où le pire peut arriver et où toute échappatoire devient dès lors impossible. La Gazette des tribunaux renforce l'idée que le couple est pris dans un lien indissoluble duquel on ne se délie que par un drame, même dans une société qui vient d'admettre le divorce.

Si on regarde ces faits divers dans un miroir, on y lira le rêve d'une société policée et ordonnée, où les passions humaines sont évitées. L'alcool, le divorce et l'union libre, présentés comme des agents destructeurs du couple et de la famille, ne devraient pas exister, suggèrent les chroniqueurs. À la jonction des deux siècles, ces discours sur le couple en rencontrent d'autres sur l'enfance maltraitée et délinquante dont sont responsables l'abus d'alcool, le divorce et l'union libre.

34. La Gazette des tribunaux, 20 septembre 1894.

35. La Gazette des Tribunaux, 3 décembre 1905, Chronique, Paris. 
RÉSUMÉ

La Gazette des Tribunaux, journal de jurisprudence et de débats judiciaires est le plus célèbre des périodiques judiciaires du xixe siècle. Au sein d'une rubrique "Chronique", il regroupe un ensemble de faits disparates qui n'ont pu trouver leur place dans les autres rubriques du quotidien constituées par les décisions de jurisprudence et les débats judiciaires de la justice civile et criminelle. La structure de la "Chronique » est restée relativement stable. Les faits divers sont placés en fin de pagination, à la troisième page du quotidien qui en compte quatre, la dernière étant constituée des annonces légales. Les drames conjugaux occupent une place non négligeable et renseignent sur les difficultés conjugales comme sur les attentes des hommes et des femmes. Mais c'est souvent un portrait sombre qui est proposé aux lecteurs. La jalousie, I'alcool, la brutalité et l'esprit de vengeance dominent dans les informations proposées et donnent l'impression que les relations entre les sexes sont toujours heurtées et prêtes à basculer dans le monde du crime.

\section{ABSTRACT}

La Gazette des Tribunaux, journal de jurisprudence et de débats judiciaires is the most famous judicial newspaper of the $19^{\text {th }}$ Century. Whitin a page called "Chronique", disparate facts which could not have been placed inside the other pages of the daily newspaper are gathered. The stucture of the "Chronique" remains relatively stable. The "faits divers" are placed at the end of the pagination, in the third page of the daily newspaper which counts four of them, the last one deals with legal ads. The marital dramas are not inconsiderable within these "faits divers" and inform about the marital difficulties and the men's and women's expectations. Yet, it is often a dismal portait which is proposed to the readers. Jealousy, alcohol, brutality and spirtit of revenge outclass in the information given in and give the impression that the relationship between the two sexes are always clashing and ready to topple over into the world of crime. 
